(S)

University.

\title{
Models of Multifunctional Central Pattern Generators: Polyrhythmic Bursting
}

Jeremy Wojcik, Robert Clewley and Andrey Shilnikov

Neuroscience Institute and Department of Mathematics and Statistics, Georgia State University, Atlanta, GA, USA

Abstract

We demonstrate a motif of three reciprocally inhibitiory cells that is able to produce multiple patterns of
bursting rhythms. Through the examination of the qualitative geometric structure of two-dimensional maps

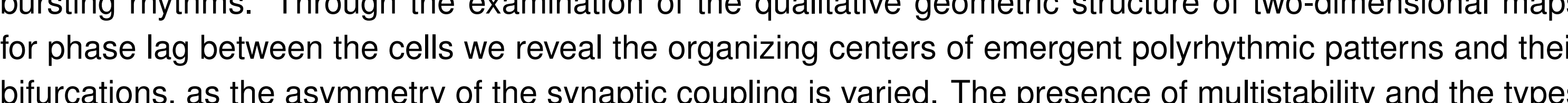
of attractors in the network are shown to be determined by the duty cycle of bursting. This analysis does not require knowledge of the equations that model the system, and so provides a powertul new approach to
studying regulatory networks. Thus, the approach is applicable to a variety of biological phenomena beyond studying regul
motor control.

\begin{tabular}{|c|}
\hline \\
\hline 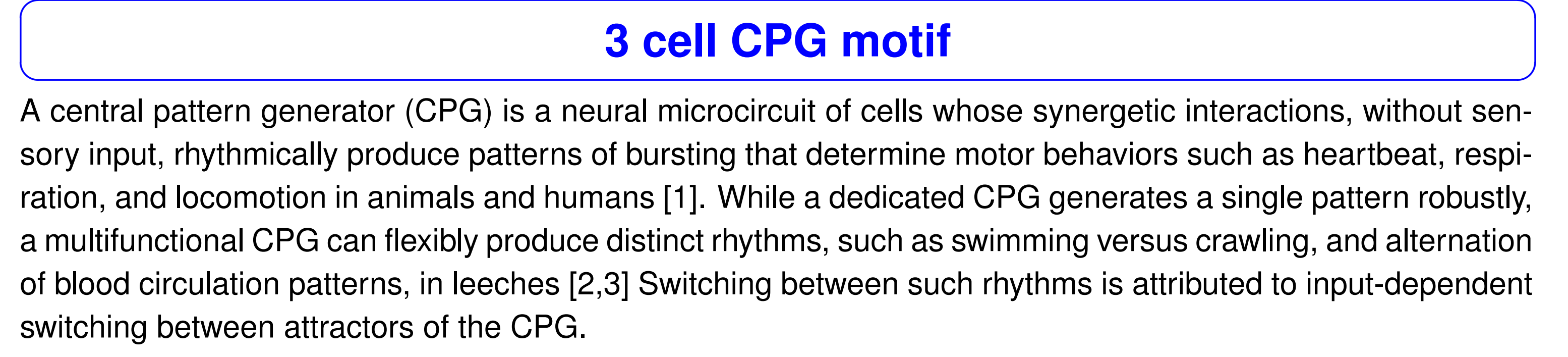 \\
\hline \\
\hline
\end{tabular}

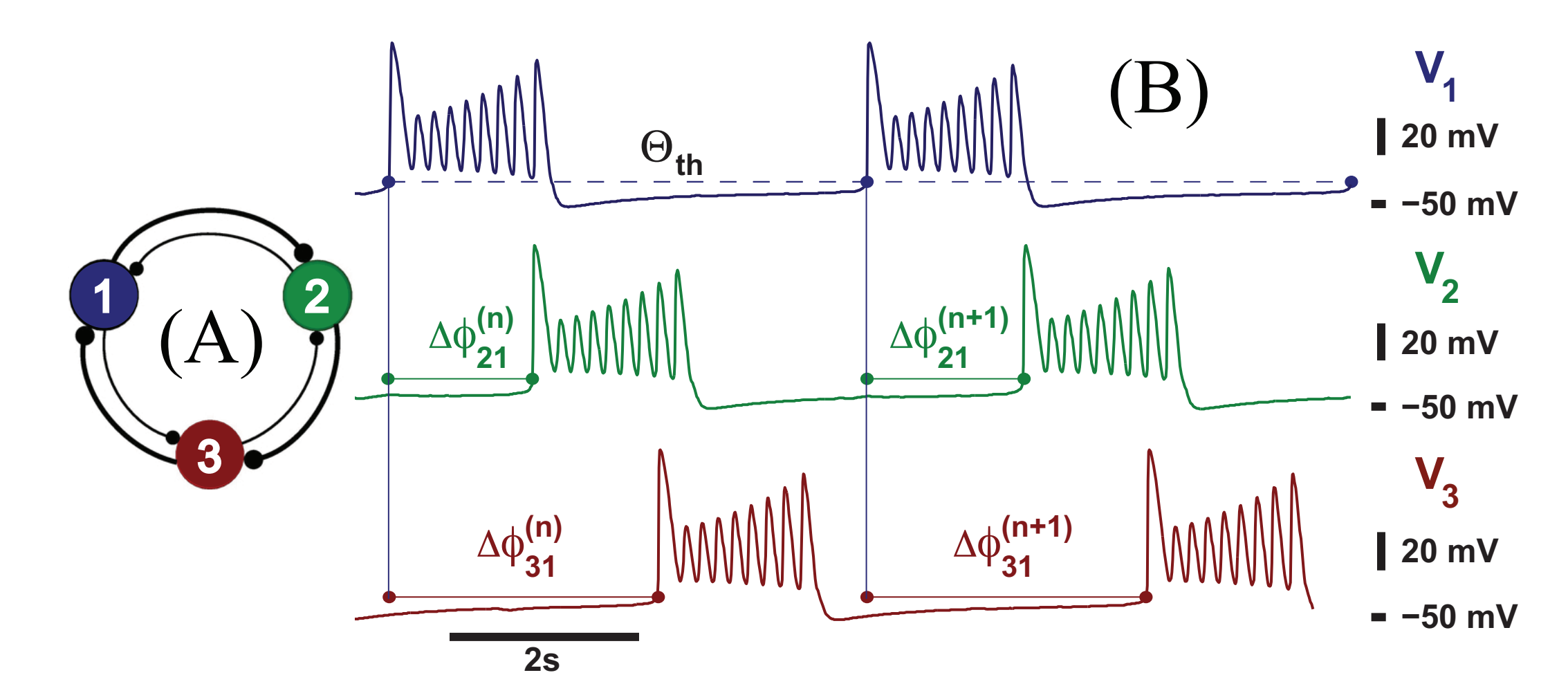

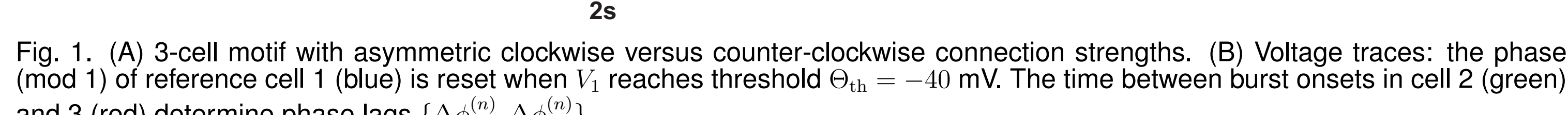
As the period of network oscillations varies over time, we derine delays between cell 2 (green) and cell 3
(sed) relative to the the referencece cell 1 (blue) at the instances the voltages $V$ increase through a thresholl of $\Theta_{\text {in }}=-40 \mathrm{mV}$, thus indicating the onset of new bursting cycles (Fig. 1). Initial delays are controlled by the

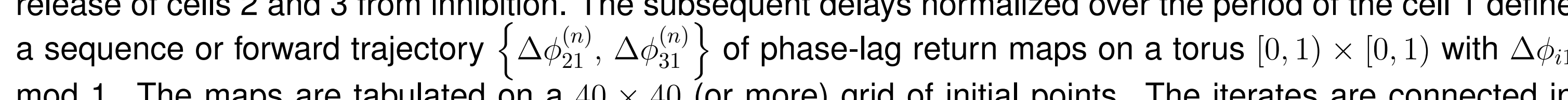
mod 1. The maps are tabulated on a $40 \times 40$ (or more) grid of initial points. The iterates are connected in
figures below to preserve order. Now we can study the dynamical properties of the maps, locate fixed point ingures below to preserve order. Now we can study the dynamical properties of the maps, locate fixed point the parameters, DC and $g_{a s}$, are varied

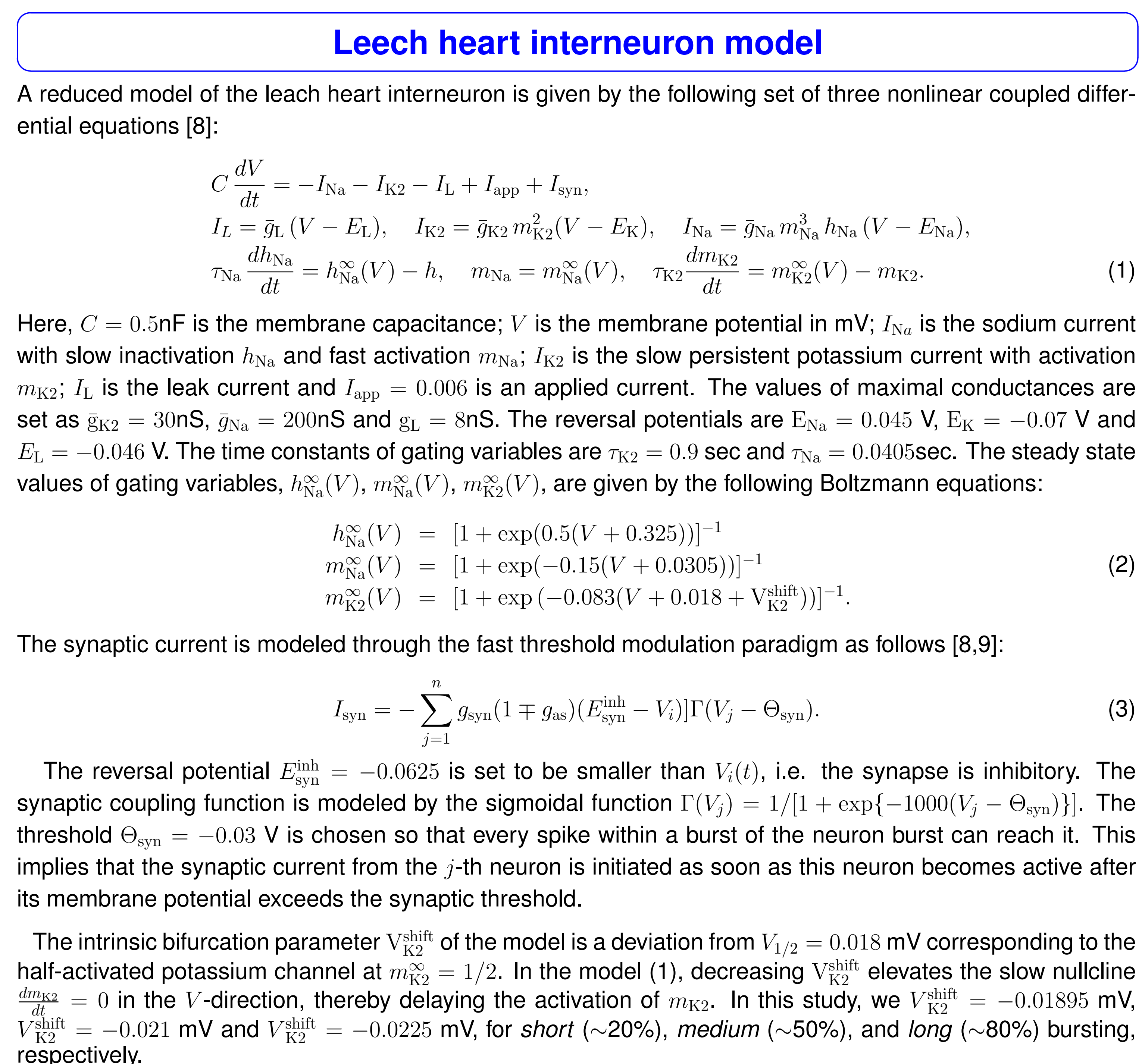

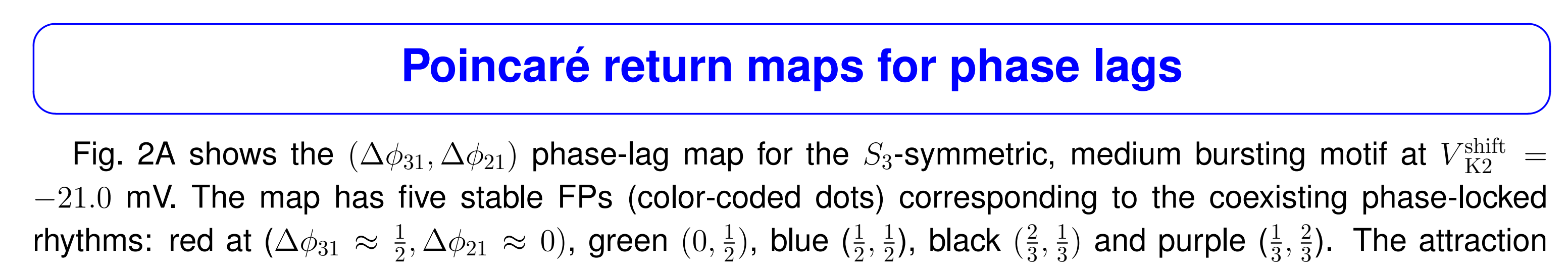

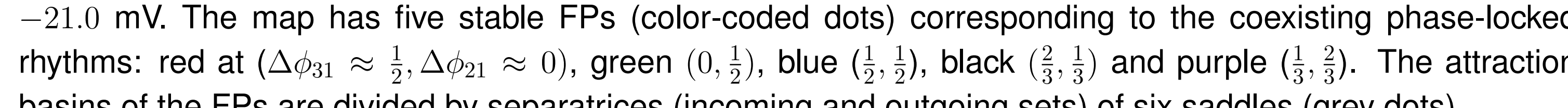

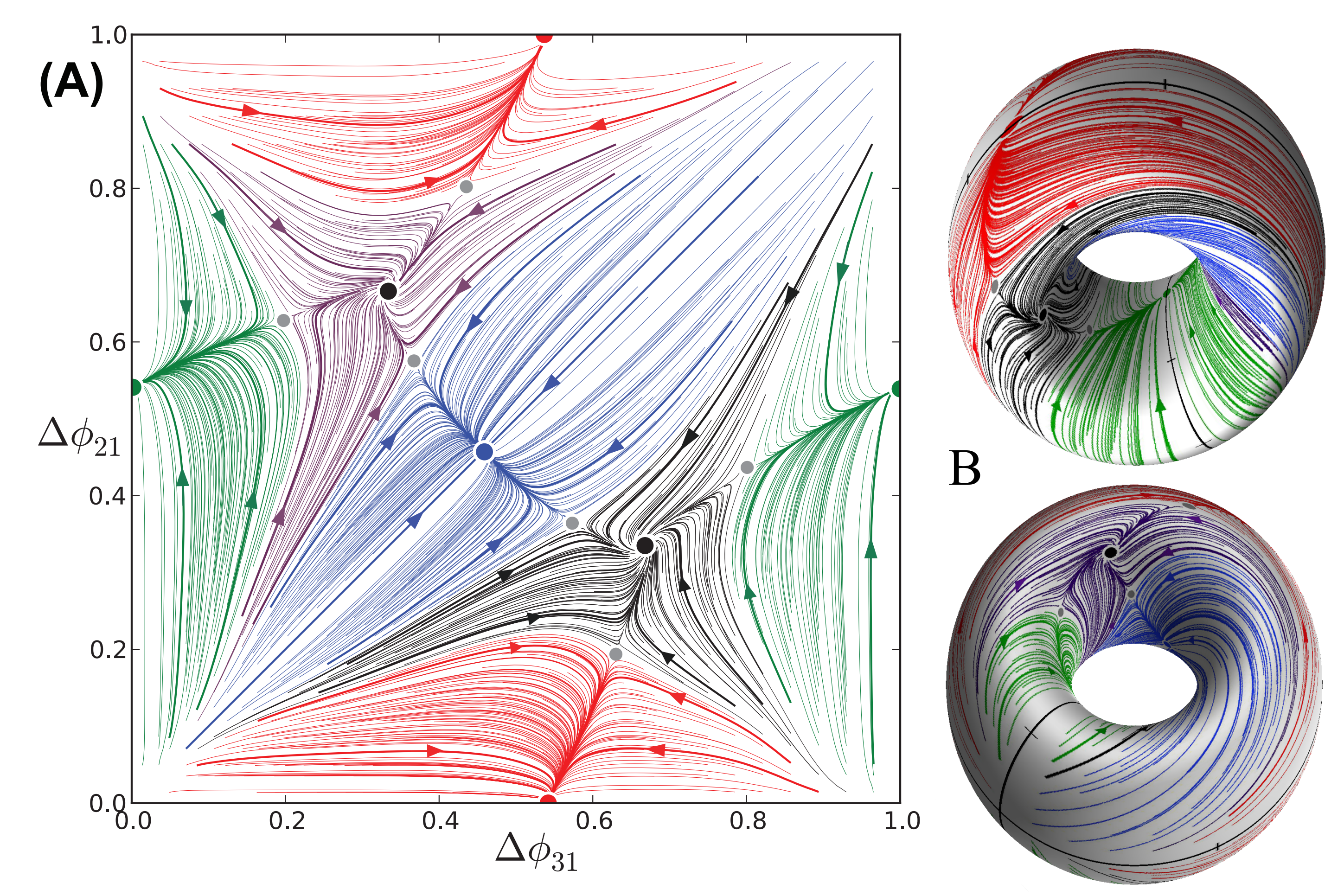

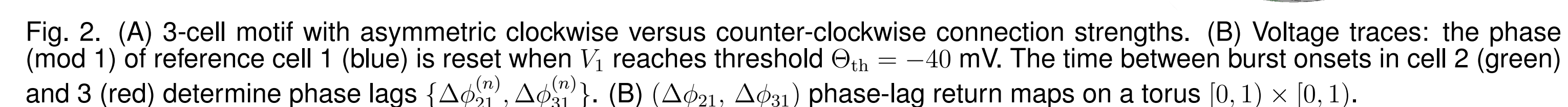
The coordinates of the FPs determine the phase locked states within bursting rhythms of the motit that are denoted symbolically as follows: $(1<2<3)$ and $(1<3<2)$ for clockwise and counter-lockwise traveling waves
of bursting (resp.) around the ring (episodes (ii) and (ii) of the voltage trace in Fig. 2) corresponding to the

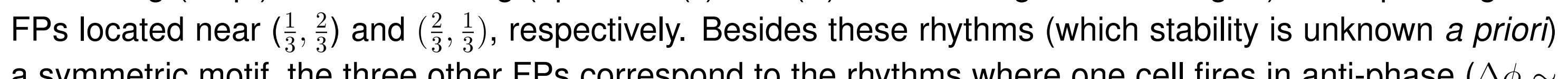

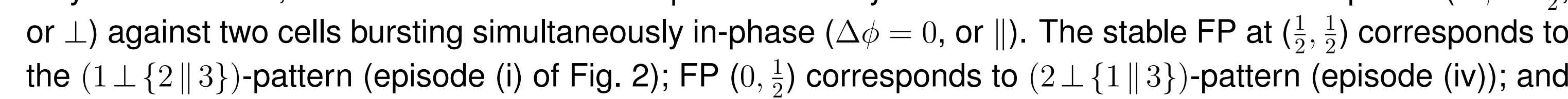

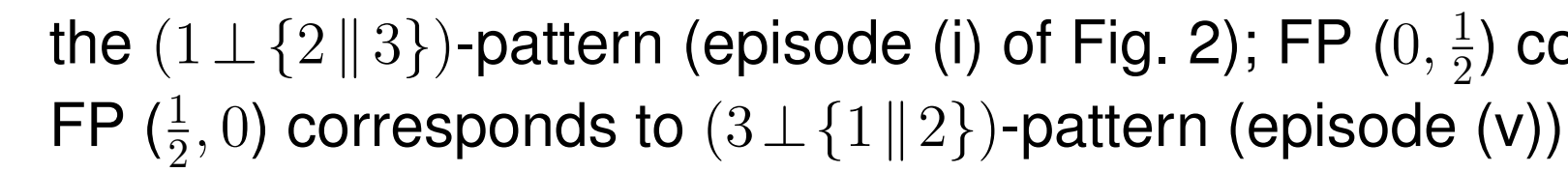

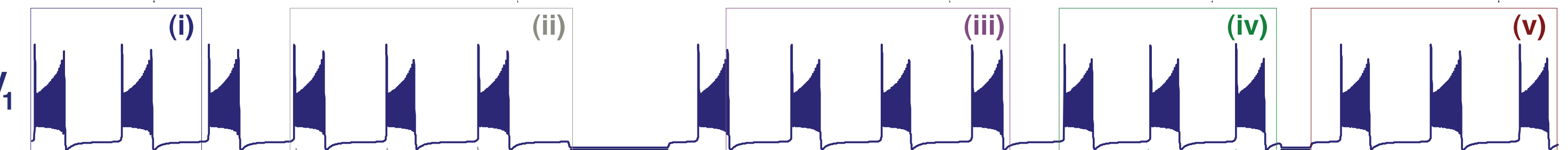

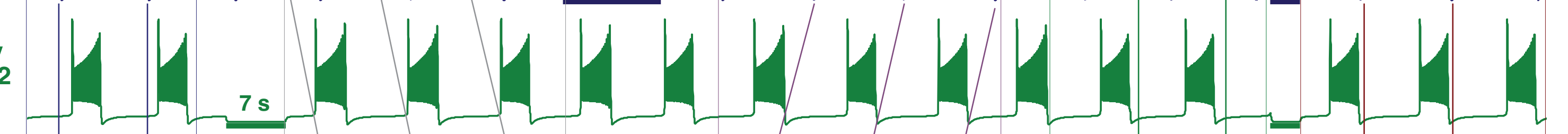

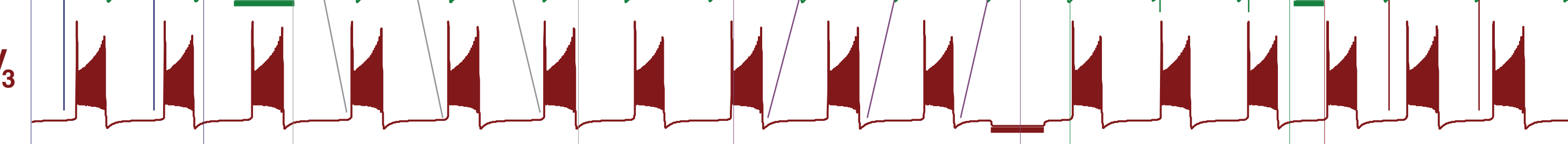

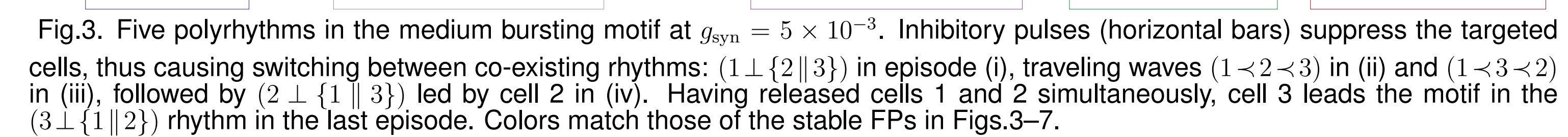

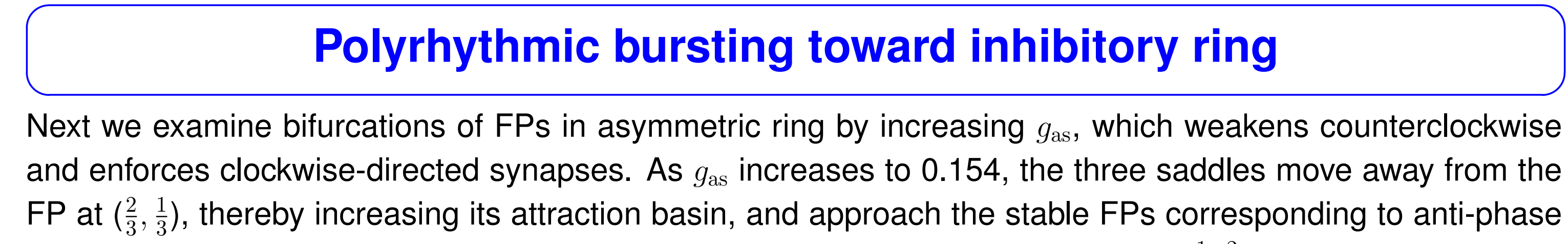

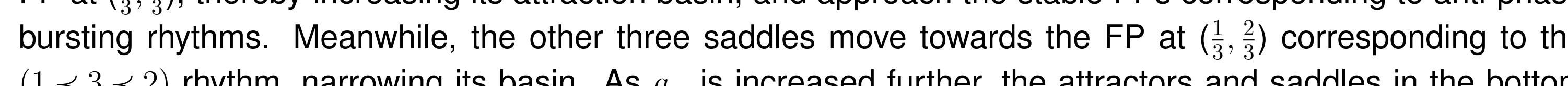

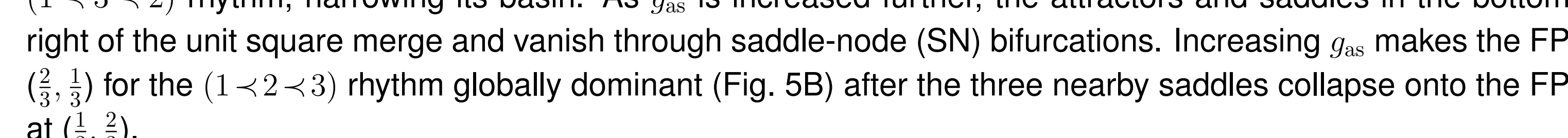
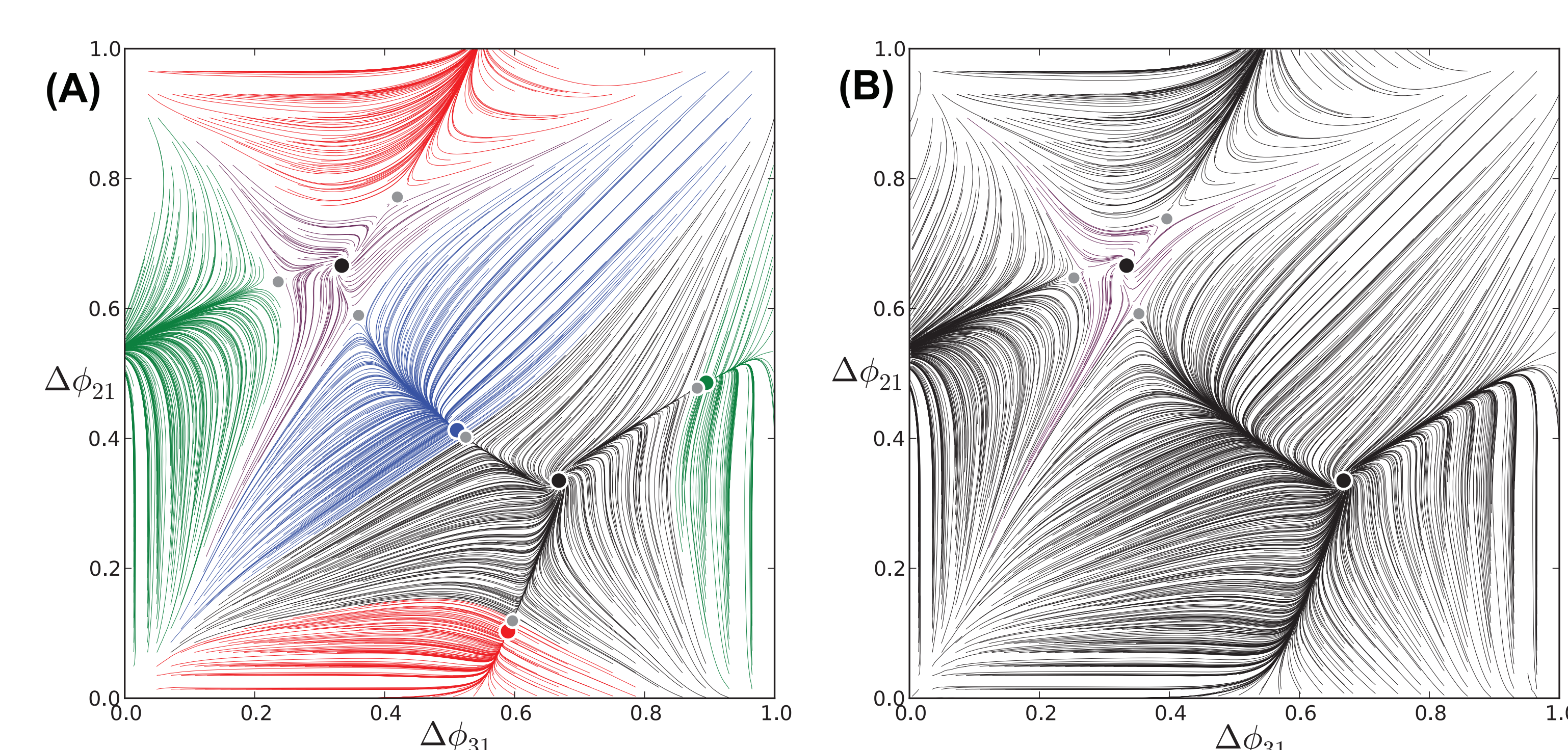

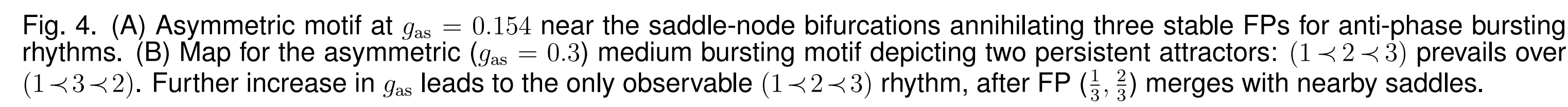
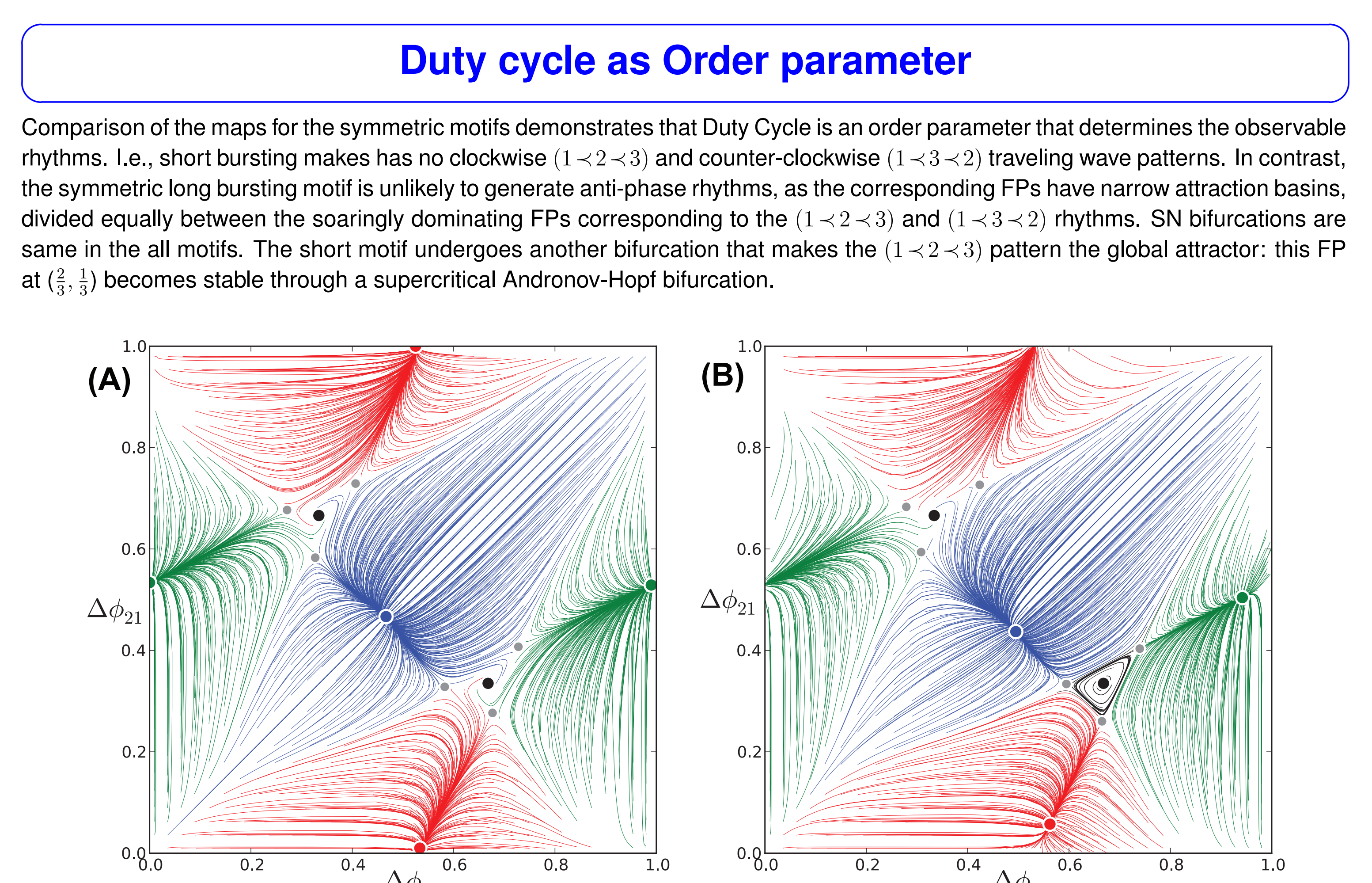

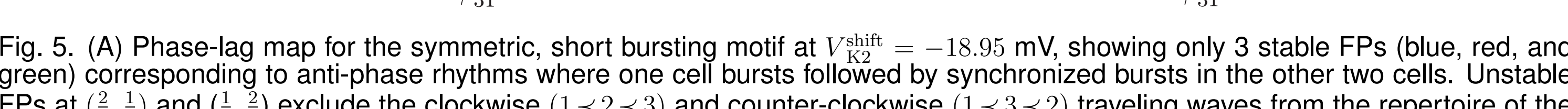

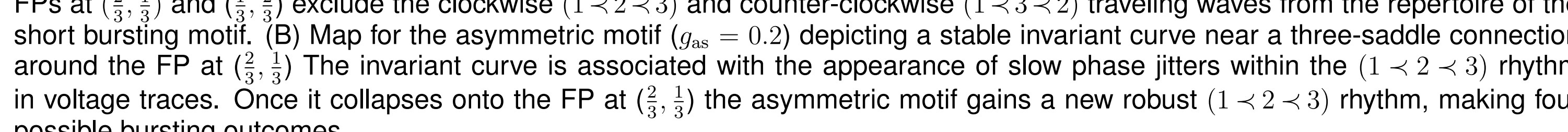
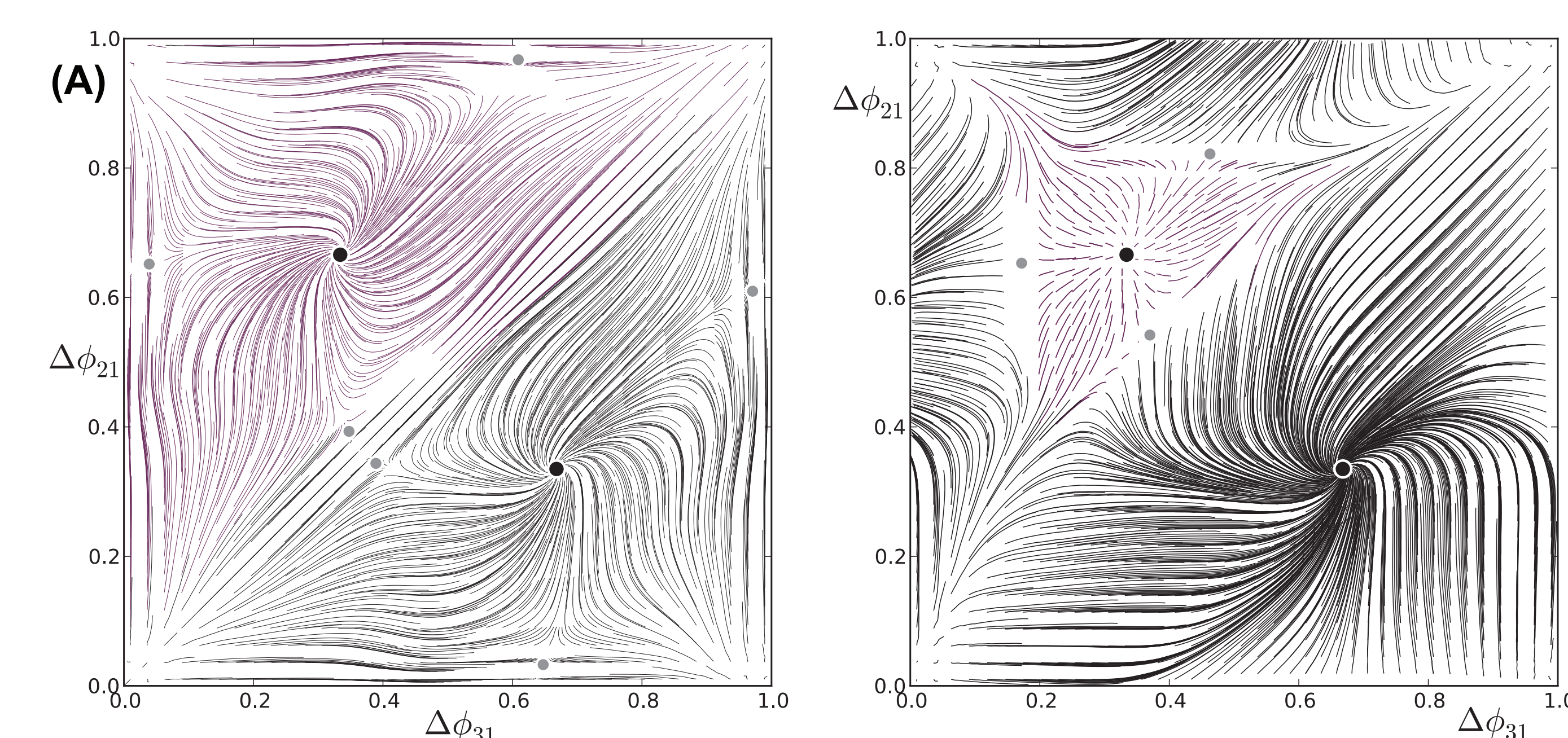

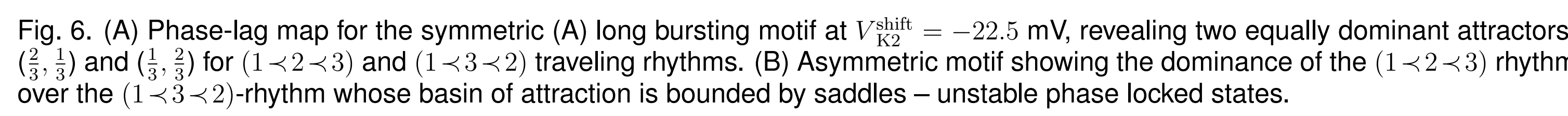

Breaking symmetry: making dedicated pacemakers
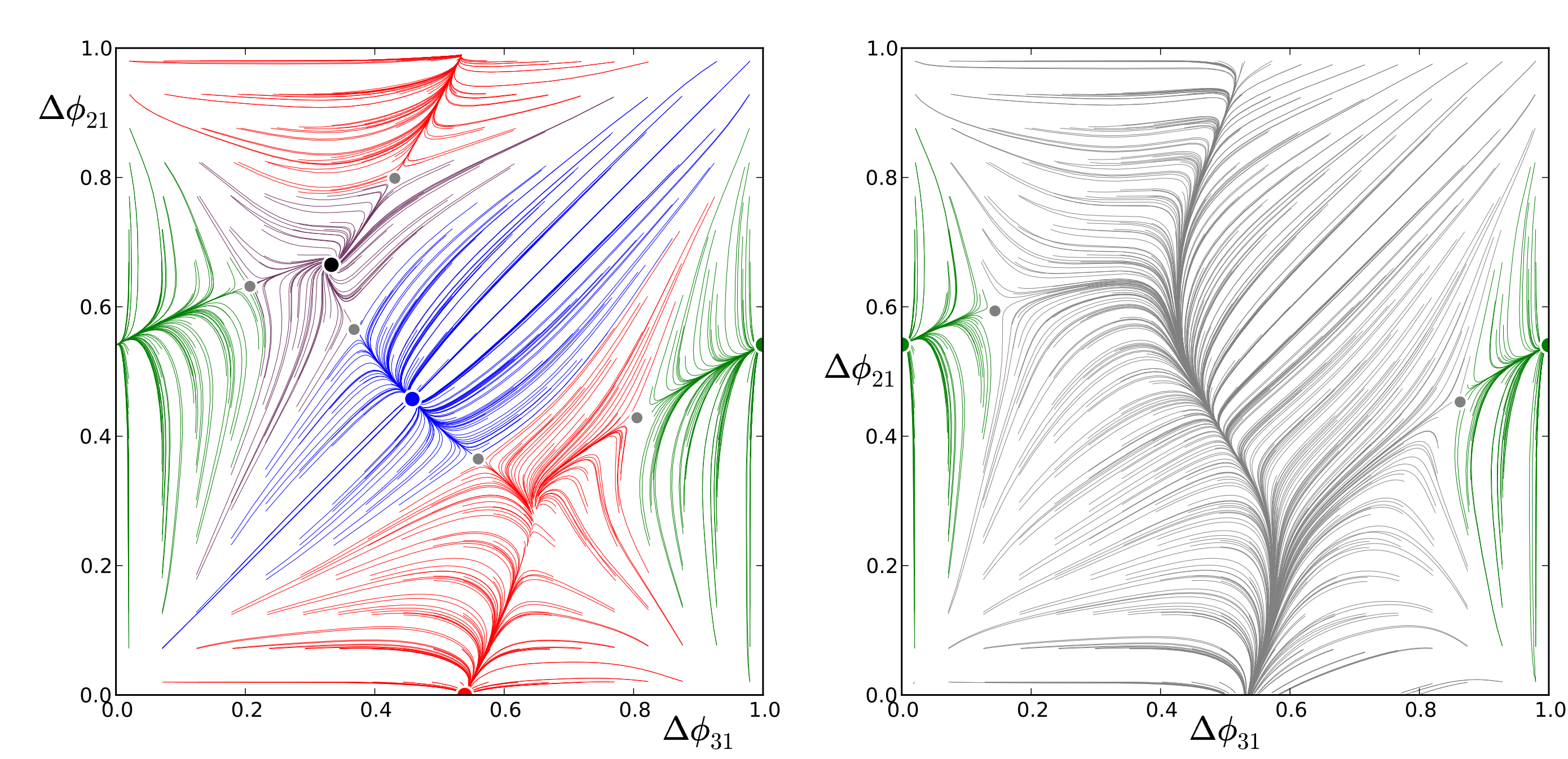

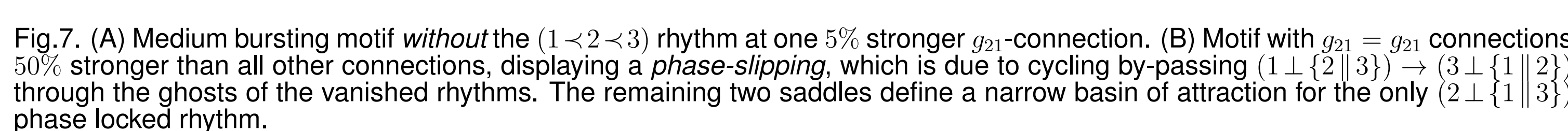
Acknowledgment We acknowledge support from NSF Grants CISE/CCF-08297422 (to R.C.), DMS-1000591 and RFFI Grant
No. 08-01-00083 (to A.S.), GSU Brains \& Behavior program, and Brain Corporation.

References

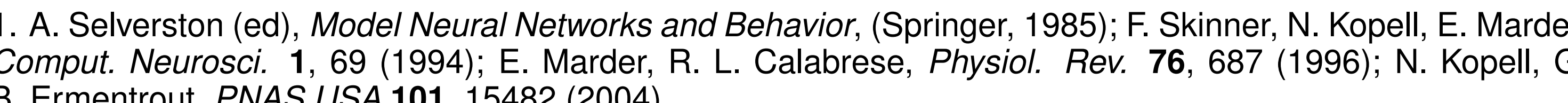

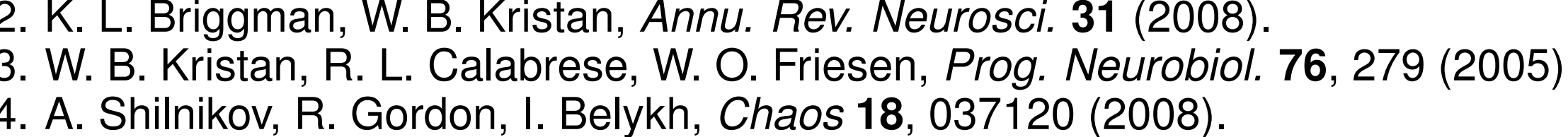

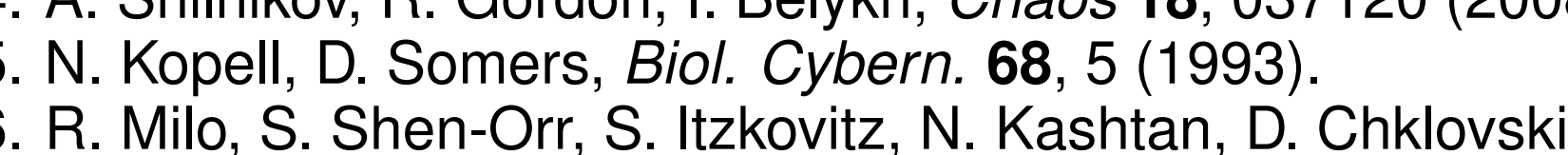

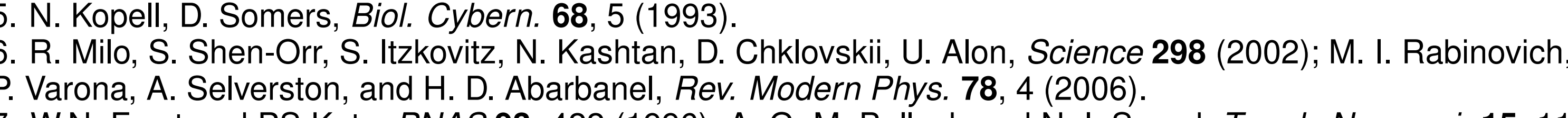
M. Bulloch and N. I. Sayed, Trends Neurosci. 15, 11

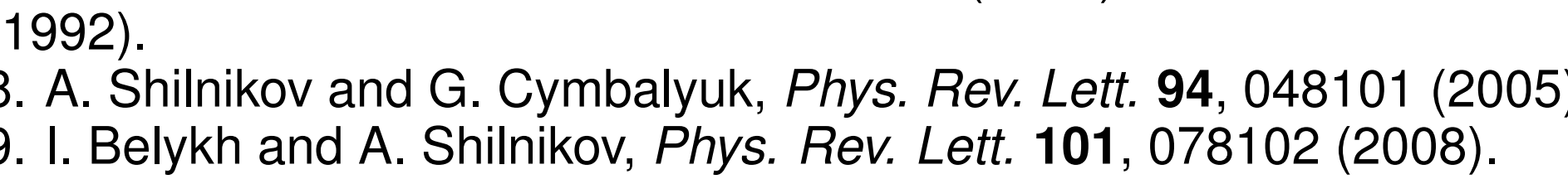

